\title{
Fingerprinting electronic structure of heme iron by $a b$ initio modeling of metal L-edge X-ray absorption spectra
}

Meiyuan Guo, ${ }^{\dagger, \ddagger}$ Erik Källman, ${ }^{\ddagger}$ Rahul V. Pinjari, ${ }^{\llbracket}$ Rafael C. Couto, ${ }^{\ddagger}$ Lasse Kragh Sørensen, ${ }^{\ddagger}$ Roland Lindh, ${ }^{\ddagger}$ Kristine Pierloot, ${ }^{\S}$ and Marcus Lundberg ${ }^{*, \neq, \|}$

$\dagger$ †epartment of Chemistry and Chemical Engineering, Southwest University, Chongqing 400715, China.

$\ddagger$ Department of Chemistry - Angström Laboratory, Uppsala University, SE-751 20 Uppsala, Sweden

ISchool of Chemical Sciences, Swami Ramanand Teerth Marathwada University, Nanded 431606, Maharashtra, India

$\S$ Department of Chemistry, University of Leuven, Celestijnenlaan 200F, B-3001 Heverlee-Leuven, Belgium.

||Department of Biotechnology, Chemistry and Pharmacy, Università di Siena, Via A. Moro 2, 53100 Siena, Italy.

E-mail: marcus.lundberg@kemi.uu.se 


\begin{abstract}
The capability of the multiconfigurational restricted active space approach to identify electronic structure from spectral fingerprints is explored by applying it to iron L-edge X-ray absorption spectroscopy (XAS) of three heme systems that represent the limiting descriptions of iron in the $\mathrm{Fe}-\mathrm{O}_{2}$ bond, ferrous and ferric $\left[\mathrm{Fe}(\mathrm{P})(\mathrm{ImH})_{2}\right]^{0 / 1+}$ $(\mathrm{P}=$ porphine, $\mathrm{ImH}=$ imidazole $)$, and $F e^{I I}(P)$. The level of agreement between experimental and simulated spectral shapes is calculated using the cosine similarity, which gives a quantitative and unbiased assignment. Further dimensions in fingerprinting are obtained from the L-edge branching ratio, the integrated absorption intensity, and the edge position. The results show how accurate ab initio simulations of metal L-edge XAS can complement calculations of relative energies to identify unknown species in chemical reactions.
\end{abstract}

\title{
Introduction
}

Reactions catalyzed by $3 \mathrm{~d}$-transition metals are important in a number of fields, including photo- and electrocatalysis, biochemistry, and materials science. ${ }^{1-5}$ Mechanisms are often described in terms of changes in oxidation state of the $3 \mathrm{~d}$ metal, and in some cases also changes in spin multiplicity or electronic state. Theoretical chemistry is frequently used to predict mechanisms of chemical reactions. In most cases, this is done by calculating relative stabilities, i.e., using the energy as the criterium to distinguish between alternative geometric and electronic structures. Reliable predictions require that the errors are smaller than the energy differences between different species. This is challenging for systems with small energy gaps, as often encountered with transition metals. The total charge of a complex, which in turn determines the metal oxidation state, can be even more difficult to assign from energy considerations as electron affinities are strongly influenced by environmental effects.

Wider acceptance of theoretically predicted mechanisms therefore often requires spectroscopic verification of key intermediates. X-ray spectroscopy, due to its elemental specificity 
and sensitivity to the local valence electronic structure, is widely used to extract oxidation and spin state of catalytic metals. ${ }^{1,3,5-9}$ Thanks to the high time resolution, X-rays can be used to study intermediates in ultrafast chemical reactions. ${ }^{10}$ Metal L-edge $(2 \mathrm{p} \rightarrow 3 \mathrm{~d}$ excitations) X-ray absorption spectroscopy (XAS) directly probes the 3d orbitals involved in metal-ligand bonding and redox reactivity. Recent developments makes it possible to apply this technique also to systems in solution and metal enzymes. ${ }^{4,11-15}$

Several observables can be used to analyze L-edge XAS spectra. The shape of the spectrum depends strongly on the electronic structure and has been used extensively to extract detailed information about metal-ligand interactions. ${ }^{16-19}$ The incident energy reflects the metal oxidation state, at least for relatively ionic complexes. ${ }^{6,8,17,20,21}$ The integrated absorption intensity is proportional to the number of holes and the metal-ligand covalency. Finally, the edge is split into $L_{3}$ and $L_{2}$ edges by $2 p$ spin-orbit coupling and the branching ratio $\left[I\left(L_{3}\right) / I\left(L_{2}+L_{3}\right)\right]$ is sensitive to the spin multiplicity in the initial state. ${ }^{22}$

Metal L-edge XAS spectra of open-shell systems are affected by both 3d-3d and 2p$3 \mathrm{~d}$ interactions, as well as $2 \mathrm{p}$ and $3 \mathrm{~d}$ spin-orbit coupling. In addition to the electrons in the open shells, there is also significant correlation with other occupied orbitals, especially metal 3s and 3p. ${ }^{23,24}$ This complicates the mapping of electronic structure to spectral shape. A standard modeling approach is the charge-transfer multiplet (CTM) method, ${ }^{25,26}$ which has also been applied to heme systems. ${ }^{18,19,27}$ It includes parameters that are fitted to the experimental spectrum, which makes it less suitable for predictive purposes. A number of models without system-specific parameters have also been used to describe metal L-edge XAS spectra of molecular systems. ${ }^{28-34}$ Multiconfigurational wavefunction approaches are well suited to treat correlation in transition metal systems, among which the complete active space is most widely used. X-ray processes typically involve only single core-orbital excitations, which makes it convenient to use a restricted active space (RAS) wavefunction. ${ }^{35-37}$ In the RAS approach, calculations can either be performed at the self-consistent field (RASSCF) or at the second-order perturbation (RASPT2) level. RAS has been successfully applied 
to simulate L-edge XAS and resonant inelastic X-ray scattering (RIXS) of several transition metal systems. ${ }^{4,30,38-43}$ However, the reliability of fingerprinting using RAS simulated spectra has not been systematically explored.

Among the different variables that are typically used to characterize L-edge XAS spectra, branching ratios, relative energies and integrated intensities are straightforward to calculate and compare. However, the quality of the predicted spectral shapes have only been determined by visual inspection, which is a subjective measure that can be affected by expectation bias. Here the accuracy of spectral shapes will instead be evaluated quantitatively using the cosine similarity (CS). This measure, the cosine of the angle between two N-dimensional vectors where $\mathrm{N}$ is the number of data points in the spectra, has previously been applied to IR and UV/Vis spectra, ${ }^{44,45}$ but this is the first application to X-ray spectra.

This study will focus on metal L-edge XAS simulations of iron porphyrins. They are cofactors in the important class of heme enzymes that include hemoglobin, cytochrome c and cytochrome P450. ${ }^{46,47}$ Experimentally, the $\pi \rightarrow \pi^{*}$ transitions in the porphyrin obscure $\mathrm{UV} /$ Vis probes of the heme iron but Fe L-edge XAS has been successfully used to probe the electronic structure of heme systems in solid, ${ }^{18,27}$ solution, ${ }^{48,49}$ and bound to surfaces. ${ }^{50,51}$ RAS simulations of heme L-edge XAS spectra have already been performed. ${ }^{52}$ These simulations targeted large models and used RASSCF with a minimum active space. Here the target is to achieve better accuracy through the use of larger active spaces and RASPT2.

The modeling approach is explored for three iron porphyrin complexes, ferrous and ferric $F e(P)(I m H)_{2}(\mathrm{P}=$ porphine, $\operatorname{ImH}=$ imidazole $)$, and $F e^{I I}(P)$, see Figure 1. The first two have well-known electronic structures and will be used to establish the accuracy of the simulations, and how to most clearly identify changes in oxidation state. Iron porphyrins that lack axial ligands typically have triplet $(S=1)$ ground states, although more than one electronic state can mix in through spin-orbit coupling. ${ }^{53-55}$ The presence of several low-lying electronic states have made $F e^{I I}(P)$ a prominent example of the challenges to reliably assign spin multiplicity from calculations of relative energies. ${ }^{24,56-71}$ The latest high-level complete 


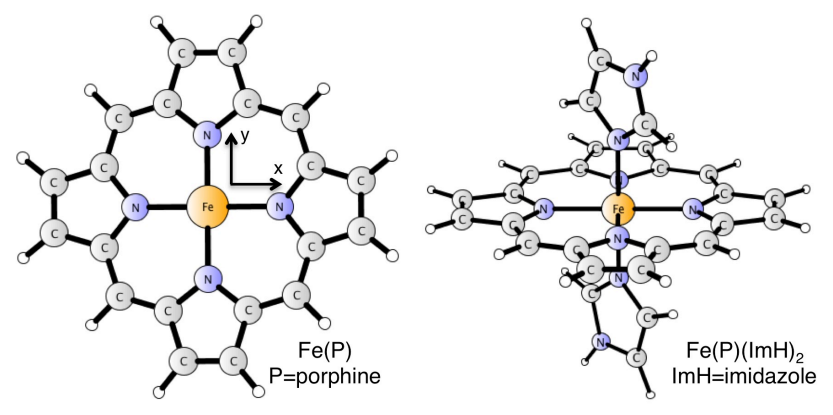

Figure 1: Structures and coordinate system for $F e^{I I}(P)$ and $\left[F e(P)(I m H)_{2}\right]^{0 / 1+}$.

active space (CAS) with second order perturbation (PT2) calculations, including coupledcluster corrections for the $3 \mathrm{~s} 3 \mathrm{p}$ correlation, predicts a triplet $0.2 \mathrm{kcal} / \mathrm{mol}$ more stable than the quintet. ${ }^{70}$ As an alternative to more and more sophisticated energy calculations, this paper will illustrate how spin state and symmetry can instead be assigned from simulations of X-ray spectra.

Together, the selected complexes represent the three limiting descriptions of iron in $\mathrm{Fe}-\mathrm{O}_{2}$ binding in hemoglobin. ${ }^{27}$ Comparing the results for all three systems will reveal how well the RAS simulations can verify the electronic structure of the $\mathrm{Fe}-\mathrm{O}_{2}$ system. At the same time it will indicate how to best use the approach to identify reaction intermediates for a wider range of transition-metal catalysts.

\section{Methods}

All experimental spectra are taken from reference. ${ }^{27}$ Data for the two six-coordinate complexes were collected for $F e^{I I}(\operatorname{TpivPP})(1-M e I m)_{2}(\operatorname{TpivPP}=\operatorname{meso-tetra}(\alpha, \alpha, \alpha, \alpha$-opivalamidophenyl)porphyrin, 1-MeIm $=1$-methylimidazole $)(S=0)$ and $\left[\mathrm{Fe}^{I I I}(\mathrm{TPP})(\operatorname{ImH})_{2}\right] \mathrm{Cl}$ $(\mathrm{TPP}=$ meso-tetraphenylporphyrin $)(S=1 / 2) \cdot{ }^{72,73}$ For the four-coordinate $F e^{I I}(P)$ complex, data for $F e^{I I}(T p i v P P)$ have been used. ${ }^{27,73}$ Although many iron porphyrins without axial ligands are triplets, ${ }^{53-55}$ the spin multiplicity of $F e^{I I}($ TpivPP) in the solid state has 
been assigned, through Mössbauer spectroscopy and magnetic moment measurements, as a quintet $(S=2) .{ }^{73}$ The quintet state is likely a result of crystal packing forces as the solvated complex has a triplet state ground state. ${ }^{74-76}$

RAS simulations were performed using a development version of MOLCAS. ${ }^{77}$ All calculations are made using the bare porphine ligand without any peripheral substituent groups, see Figure 1. This is motivated by the very small differences betwen $F e^{I I}(T P P)(\operatorname{ImH})_{2}$ and $\mathrm{Fe}^{\mathrm{II}}(\mathrm{TpivPP})(1-\mathrm{MeIm})_{2}$ L-edge XAS spectra. ${ }^{27}$ Detailed computational comparisons of the electronic structure of metal porphine and metal tetra-phenyl porphyrins showed that electron affinities change by only $0.2 \mathrm{eV}$, while the relative energy of valence orbitals are not affected. ${ }^{78}$ The substituent effects on iron-centered X-ray transitions should therefore be small. Geometries were optimized using a mixture of DFT and CASPT2 methods. For details, see Section 1 of the Supporting Information (SI). Deviations in iron-ligand distances compared to experiment are less than $0.02 \AA$, and these deviations should only have minor effects on the calculated spectra. ${ }^{79}$ The $\mathrm{Fe}(\mathrm{P})(\mathrm{ImH})_{2}$ complexes belong to the $C_{2 h}$ point group. The $F e^{I I}(P)$ complex to the $D_{4 h}$ point group, although calculations are performed using the Abelian $D_{2 h}$ point group.

For all active spaces, the iron $2 \mathrm{p}$ orbitals are included in RAS1 allowing up to one excitation. The core orbitals are the only ones in the active space with ungerade symmetry, which makes it easy to generate core-excited states as they are the lowest states in the ungerade irreducible representations. To avoid orbital rotation, i.e., that the hole appears in a higher-lying orbital, the $2 \mathrm{p}$ orbitals have been frozen in the final states.

For $F e^{I I}(P)(I m H)_{2}$ the RAS2 space was systematically designed, starting from a minimal active space with only the five metal 3d-dominated orbitals, see Figure 2. For simplicity, these orbitals will be labeled $t_{2 g}$ and $e_{g}$ using the well-known $O_{h}$ point group nomenclature, to which the complexes approximately belong. From the minimal active space, two filled ligand-dominated $\sigma$ bonding orbitals are included, one in the porphyrin plane and one along the imidazole axis, as they correlate strongly with the empty anti-bonding $e_{g}$ orbitals. The 


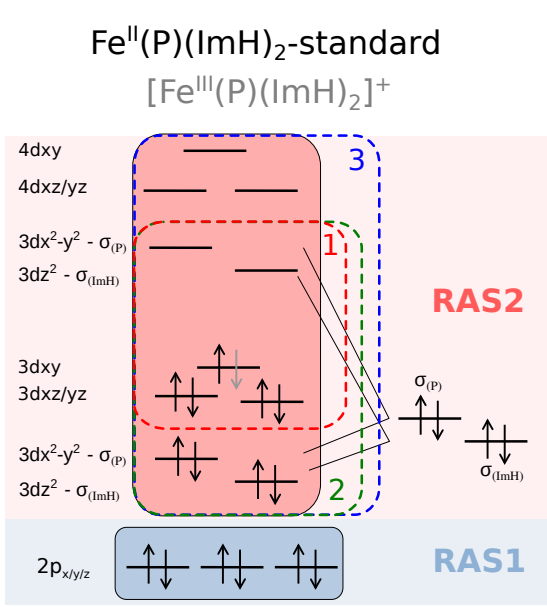

\section{$\mathrm{Fe}^{\prime \prime}(\mathrm{P})(\mathrm{ImH})_{2}$-alternative}

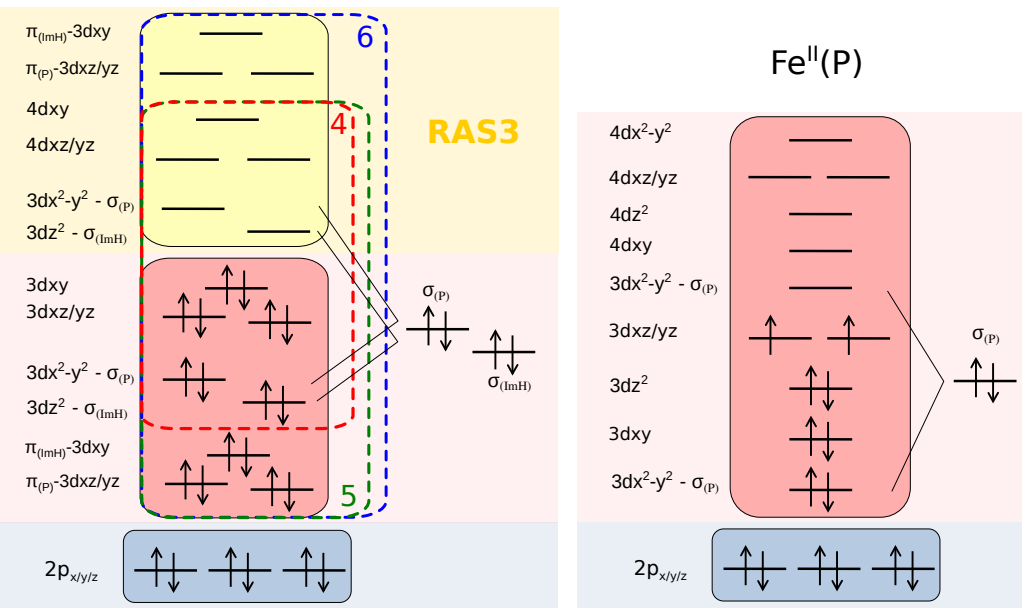

Figure 2: Schematic illustrations of the RAS active spaces. The standard active space of $\mathrm{Fe}^{I I}(\mathrm{P})(\mathrm{ImH})_{2}$ and $\left[\mathrm{Fe}^{I I I}(\mathrm{P})(\mathrm{ImH})_{2}\right]^{+}$(one electron less) is shown to the left with: (1) $\operatorname{RAS}(12,1,0 ; 3,5,0)$, (2) RAS $(16,1,0 ; 3,7,0)$, (3) $\operatorname{RAS}(16,1,0 ; 3,10,0))$. The alternative active spaces of $F e^{I I}(P)(I m H)_{2}$ with virtual orbitals in RAS3, allowing up to three excitations, in the middle: (4) RAS $(16,1,3 ; 3,5,5)$ and (5) $\operatorname{RAS}(22,1,3 ; 3,8,5)$ and (6) $\operatorname{RAS}(22,1,3 ; 3,8,8)$. The active space of $F e^{I I}(P) \operatorname{RAS}(14,1,0 ; 3,11,0)$ is shown to the right. Renderings of valence orbitals are shown in SI Section 2.

next step is to include three empty orbitals that can correlate with the filled $t_{2 g}$ orbitals. These are, unless specifically commented, metal 4d orbitals that describe the double-shell effect. ${ }^{59}$ These three active spaces are shown as 1-3 in Figure 2. RAS active spaces are systematically labeled as $\operatorname{RAS}(n, l, m ; i, j, k)$, where $i, j$, and $k$ are the number of orbitals in RAS1, RAS2, and RAS3 spaces respectively, $n$ is the total number of electrons in the active space, $l$ the maximum number of holes allowed in RAS1, and $m$ the maximum number of electrons in RAS3. In this nomenclature, these active spaces are RAS $(12,1,0 ; 3,5,0)$, $\operatorname{RAS}(16,1,0 ; 3,7,0)$, and $\operatorname{RAS}(16,1,0 ; 3,10,0)$.

To be able to further extend the size of the active space, alternative active spaces were constructed with the virtual orbitals in the RAS3 space allowing up to three electrons, one from the core-hole excitation and two more to describe electron correlation, see Figure 2. The smallest of the alternative spaces has the same orbitals as the standard RAS $(16,1,0 ; 3,10,0)$ to test the effects of the RAS3 approximation. In the next step, three filled ligand $\pi$ orbitals are added to the active space. This choice was influenced by CTM simulations of 
$F e^{I I}(P)(I m H)_{2}$, which show that the inclusion of $\pi$-donating orbitals are important to reproduce spectral shape. ${ }^{18}$ Finally, three empty orbitals were added to describe correlation among the ligand-dominated orbitals. These three active spaces are shown as 4-6 in Figure 2 and are formally written as $\operatorname{RAS}(16,1,3 ; 3,5,5), \operatorname{RAS}(22,1,3 ; 3,8,5)$, and $\operatorname{RAS}(22,1,3 ; 3,8,8)$.

Calculations of $\mathrm{Fe}^{I I I}(\mathrm{P})(\mathrm{ImH})_{2}$ have only been performed using the standard active space with ten valence orbitals, which gives $\operatorname{RAS}(15,1,0 ; 3,10,0)$. The design of the $F e^{I I}(P)$ active space follows the same principle. In addition to the five metal 3d-dominated orbitals, one porphyrin-dominated $\sigma$ bonding orbital is included to correlate with the $\sigma$-antibonding $3 d_{x 2-y 2}$ orbital. In the quintet $(S=2)$ state, all five 3 d orbitals are at least partially occupied, and therefore five additional correlating orbitals were added to the active space. This gives a total of eleven valence orbitals and a $\operatorname{RAS}(14,1,0 ; 3,11,0)$ active space, see Figure 2.

Orbital optimizations were performed using state-average RASSCF, performed separately for each spin multiplicity and irreducible representation. To select relevant spin multiplicities, the spin-selection rules of the electric dipole transitions $(\Delta S=0)$ and the selection rules for the spin-orbit operator $(\Delta S=0, \pm 1)$ were considered. For $F e^{I I}(P)(\operatorname{ImH})_{2}$, which has a singlet ground state, singlet and triplet final states were included. For $F e^{I I I}(P)(\operatorname{ImH})_{2}$, which has a doublet ground state, doublet and quartet final states were included. The contribution from higher multiplicities through coupling with the intermediate spin states are relatively small. ${ }^{21,79}$ For $F e^{I I}(P)$, where both triplet and quintet initial states were considered, singlet, triplet and quintet final states were included. Here the contribution from septets are small because they appear at lower energy than other states and do not mix strongly. ${ }^{21,22}$

Due to the wide energy range of the L-edge XAS spectra, coupled with a high density of states, a very large number of final states are required. At the same time, adding too many states can negatively affect the shape of the active orbitals. This makes it difficult to determine an optimum number of final states. Instead, the shape of the spectra as a function of the number of final states have been monitored. A detailed listing of the number 
of states is given in SI Section 3. Unless otherwise stated, results are presented for the largest number of states. For $F e^{I I}(P)(\operatorname{ImH})_{2}$ this means 120 states each in $a_{u}$ and $b_{u}$ for the twp spin multiplicities, which gives a total of 480 final states. For $\left[\mathrm{Fe}^{I I I}(\mathrm{P})(\mathrm{ImH})_{2}\right]^{+}$, the corresponding numbers are 80 and 360. For $F e^{I I}(P)$, all quintet final states were used, 17 in $a_{u}, 12$ in $b_{1 u}, b_{2 u}$ and $b_{3 u}$, together with 60 singlet and triplet final states in each irreducible representation for a total of 533 states. The orbitals in the active space do not change with the number of states, with the exception of $F e^{I I}(P)(\operatorname{ImH})_{2}$ where one or more ligand-dominated anti-bonding $\pi^{*}$ orbital enters the active space when 80 or more final states per irreducible representation are used, similar to previous observation for the iron hexacyanides. ${ }^{79}$ For this system, the orbital rotation did not lead to any significant changes in spectral shape, but the changes in the active space with the number of states illustrate the complexity of the state-average procedure.

Final energies were obtained with multistate RASPT2 including all states from the RASSCF calculations. ${ }^{37}$ In the PT2 step, no excitations from core orbitals have been included, with the exception of the iron $2 \mathrm{p}$ orbitals. To reduce problems with intruder states an imaginary shift of 0.3 hartree has been applied throughout. ${ }^{80}$ The default ionizationpotential electron-affinity (IPEA) shift of 0.25 hartree has been used ${ }^{81}$ In transition metal complexes, including hemes, the value of the IPEA shift quite strongly affects the relative energies of different states, in particular states with different spin. ${ }^{68,82}$ As there is no consensus on the optimal value of this shift, ${ }^{82,83}$ the effect of varying the IPEA shift from 0.0 to 0.6 has been tested for the standard active space of $\mathrm{Fe}^{I I}(\mathrm{P})(\mathrm{ImH})_{2}$. This gave only minor changes in absolute energy and spectral shape, see SI Section 5.1. This is consistent with previous experience that for systems with no major differences between RASSCF and RASPT2 spectra, as is the case for all three systems discussed here (see SI Sections 5.15.3), changing IPEA does not strongly affect the relative dynamic correlation contribution, which is anyway small. ${ }^{79}$ For $F e^{I I}(P)(\operatorname{ImH})_{2}$ the only visible difference is a small shift to higher energies of the second peak in the $L_{3}$ edge with larger IPEA shift. Increasing IPEA 
will systematically destabilize states with a higher spin with respect to states with a lower spin, which suggests that the second peak has a relatively large contribution from triplet final states. The value of the IPEA shift does affect the relative energies of different spin states of $F e^{I I}(P) .{ }^{68}$ Fortunately, in the current fingerprinting approach the initial state is not determined by calculated relative energies, which reduces the sensitivity to the IPEA value.

Scalar relativistic effects have been included by using a second-order Douglas-Kroll-Hess Hamiltonian, ${ }^{84,85}$ in combination with the ANO-RCC basis set and the use of a Cholesky decomposition approach to approximate the two-electron integrals. ${ }^{86-88}$ Due to the cost associated with calculating a large number of core-excited states, most calculations have been performed with the ANO-RCC-VDZP basis set. This is considerably smaller than in previous high-level energy calculations, ${ }^{68}$ but previous tests have shown the dependence of spectral shape on the size of the basis set to be relatively small. ${ }^{79}$ Calculations of $F e^{I I}(P)$ spectra using the larger ANO-RCC-VTZP show only minor effects on spectral shape, see SI Section 5.3.

Spin-orbit coupling is included by a RAS state-interaction (RASSI) approach. ${ }^{89,90}$ The RASSI method is also used to calculate electric dipole oscillator strengths in the length representation. In limited testing of transition metal L-edges, differences between length and momentum representation were minor for spectral shape and branching ratio (less than 0.01). Final spectra are generated using a Lorentzian broadening with a full-width-at-halfmaximum (FWHM) of 0.4 and $0.8 \mathrm{eV}$ for the $L_{3}$ and $L_{2}$ edges respectively, and convoluted with an experimental Gaussian broadening of $0.4 \mathrm{eV}$.

The similarity between experimental and calculated spectral shapes are evaluated using CS:

$$
\text { similarity }=\cos (\theta)=\frac{\mathbf{A} \cdot \mathbf{B}}{\|\mathbf{A}\|\|\mathbf{B}\|}=\frac{\sum_{i=1}^{N} A_{i} B_{i}}{\sqrt{\sum_{i=1}^{N} A_{i}^{2}} \sqrt{\sum_{i=1}^{N} B_{i}^{2}}}
$$


where $A_{i}$ and $B_{i}$ are components of vector $\mathbf{A}$ and $\mathbf{B}$ respectively. For vectors without negative values, the CS is between 0 and 1, with the latter value for perfect agreement. As the the angle between two vectors is independent of their length, CS scores are independent of any intensity scaling factors applied uniformly across the entire spectrum, which makes them easily transferrable between data sets. A key limitation of the CS is that the measure cannot handle peaks that are shifted in energy. The current RAS procedure typically underestimates the $L_{3}-L_{2}$ split by $\sim 1 \mathrm{eV}{ }^{41}$ A tentative explanation is that by not allowing for the core orbital relaxation in the final states, the radial extension is too large, which in turn leads to an underestimation of the spin-orbit coupling. It could also be a problem of the LS coupling scheme, because it does not allow differences in the spatial extension of the $j=3 / 2$ and $j=1 / 2$ holes. The error means that the $L_{3}$ and $L_{2}$ edges cannot be evaluated at the same time. As the $L_{3}$ edge is better resolved and easier to converge than the $L_{2},{ }^{79}$ the CS has only been evaluated over the $L_{3}$ edge (702.5-715.0 eV). The incident energies of the simulated spectra are shifted to maximize the CS and, unless otherwise specified, absorption intensities are scaled to match the integrated experimental intensity over the full edge. Scaling factors and energy shifts for all simulations are given in SI Section 3.

\section{Results}

In this section we will analyse the accuracy of the RASPT2 approach for simulating Ledge XAS spectra of the three target molecules. In the first part, the spectra of the two $\left[\mathrm{Fe}(\mathrm{P})(\mathrm{ImH})_{2}\right]^{0 / 1+}$ complexes will be analyzed to establish the expected accuracy of the simulations. In the second part, different electronic states of $F e^{I I}(P)$ will be calculated to show how the simulations can be used to separate states of different symmetry and spin multiplicity. 


\section{Fingerprints of oxidation states}

$F e^{I I}(P)(I m H)_{2}$ is a low-spin $d^{6}$ complex with a singlet $(S=0)$ ground state. The experimental XAS spectrum has a relatively sharp main $L_{3}$ peak with a maximum at $708.2 \mathrm{eV}$ associated with excitations to the $e_{g}$ orbitals $\left(O_{h}\right.$ symmetry label), see Figure 3 . On the low-energy side there are shoulders at 706 and $707 \mathrm{eV}$ and there is a small high-energy peak at $710.1 \mathrm{eV}$. The $L_{2}$ peak is at $720.1 \mathrm{eV}$ and has a broad high-energy shoulder around 722 $\mathrm{eV}$.
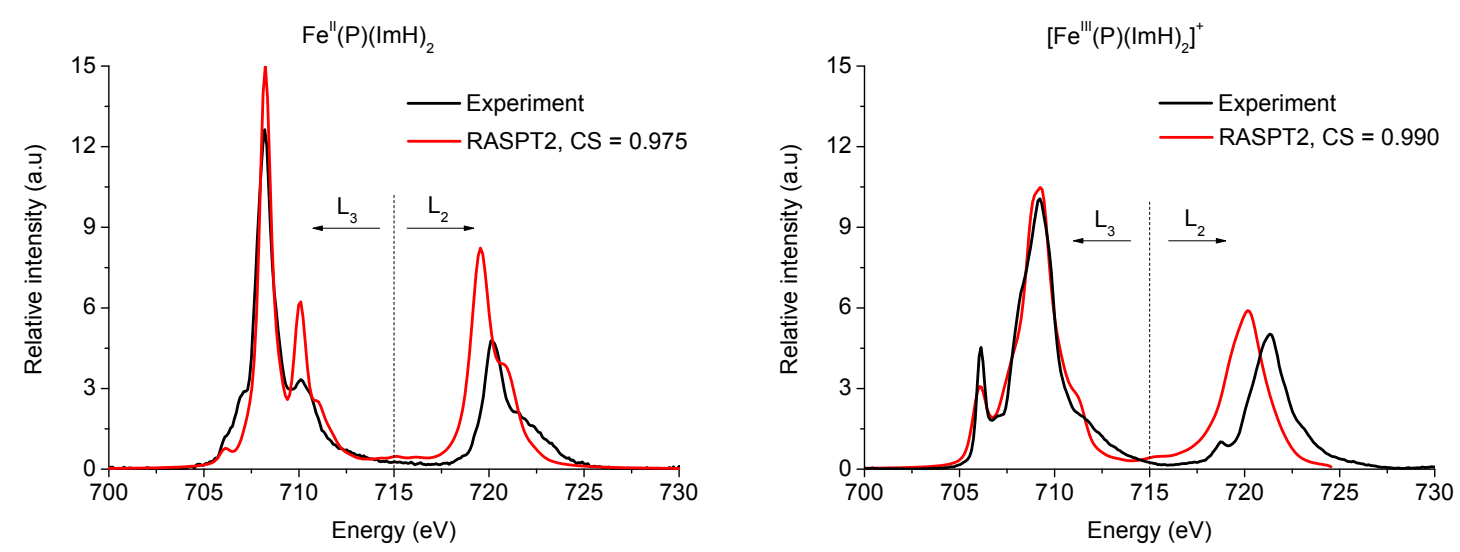

Figure 3: Experimental and RASPT2 calculated spectra of $F e^{I I}(P)(\operatorname{ImH})_{2}$ (left) and $\left[F e^{I I I}(P)(I m H)_{2}\right]^{+}$(right) with cosine similarity (CS) scores.

The RASPT2 simulation using the standard RAS $(16,1,0 ; 3,10,0)$ active space reproduces the major spectral features, the narrow main peak in the $L_{3}$ edge, as well as the minor peak at the high-energy side, although the relative intensity of the latter is overestimated, see Figure 3. The simulations also underestimate the intensity in the low-energy region. Similar to previous experience, the position of the $L_{2}$ edge is underestimated by $\sim 1 \mathrm{eV} .{ }^{41}$ The standard active space gives a CS score of 0.975 with no clear improvement in the similarity with more states or when adding PT2, see SI Section 5.1.

The results are strongly affected by the choice of active space. The similarity increases significantly when using ten valence orbitals compared to the smaller active spaces with only five or seven valence orbitals, see Figure 4. This is due to a larger overestimation of the intensity of the second peak of the $L_{3}$ edge for the smaller active spaces. The differences 
can be rationalized through an analysis of the changes in the $e_{g}$ and $t_{2 g}$ orbital occupations upon core excitation, see SI Section 5.1. In the larger active space, the major peak can essentially be assigned to a $2 p \rightarrow e_{g}$ transition, while the second peak is dominated by states with additional $t_{2 g} \rightarrow e_{g}$ excitations. In the smaller active spaces the $t_{2 g}$ electrons cannot correlate properly, which leads to an overestimation of the mixing between the main $t_{2 g}^{6} e_{g}^{1}$ configuration and highly excited configurations. This mixing leads to more equal intensity of the two peaks and this translates to a worse agreement with experiment. From Figure 4 it is thus clear that a larger active space gives more accurate results.

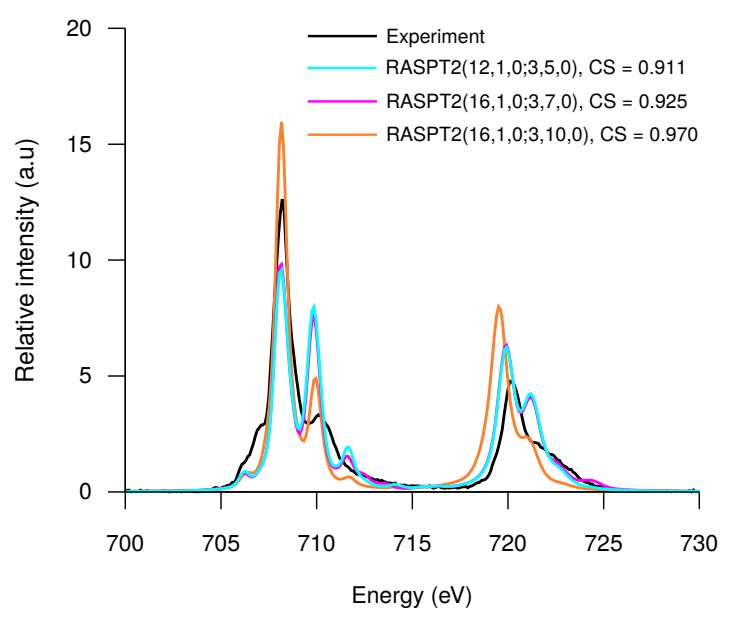

Figure 4: Effect of the active-space selection on the simulated of $\mathrm{Fe}^{I I}(\mathrm{P})(\mathrm{ImH})_{2}$. Notice that this comparison is performed using 60 instead of 120 final states per spin multiplicity and irreducible representation.

Due to the restrictions in the number of excitations, the alternative active spaces with virtual orbitals in RAS3 can be extended with even more valence orbitals, see Figure 4. However, a control using RAS3 and the same ten valence orbitals as in the standard active space, did not reproduce the spectrum obtained when including all valence excitations. Interestingly, the agreement with experiment improved giving a CS score of 0.990. This is because weak transitions in the low-energy side of the spectrum appear and the relative intensity of the minor peak improves further, see SI section 5.1. The RAS $(22,1,3: 3,8,5)$ space, which includes additional filled ligand orbitals shows even better agreement with experiment (CS $=0.992$ ), while extra empty orbitals did have the desired porphyrin character and also did 
not lead to any visible changes in the spectrum. As it is possible that the high similarity of the alternative active spaces is partly due to artefacts from the division of the valence space, results from the standard active space will be used in all further comparisons.

The experimental branching ratio of $F e^{I I}(P)(\operatorname{ImH})_{2}$ is 0.68 , see Figure $5 .{ }^{27}$ This is close to the statistical branching ratio of $2 / 3$ from the number of degenerate states in the $L_{3}$ ( $j=3 / 2,4 m_{j}$ states $)$ and $L_{2}\left(j=1 / 2,2 m_{j}\right.$ states $)$ edges. The statistical ratio only holds in the absence of both spin-orbit coupling in the initial state and electrostatic interactions between the core hole and the valence electrons in the final states. ${ }^{22}$ As $F e^{I I}(P)(\operatorname{ImH})_{2}$ lacks spin-orbit coupling in the ground state, the small deviation from $2 / 3$ is due to electrostatic interactions in the final state. The RASPT2 calculations give a significantly smaller branching ratio (0.58), which is seen in the spectrum as an overestimation of the $L_{2}$ intensity, see Figure 3.

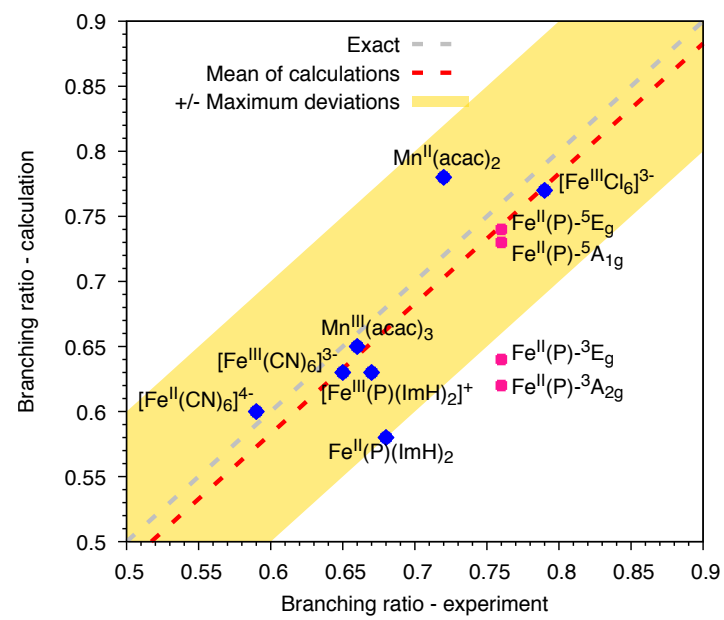

Figure 5: Experimental and RASPT2-calculated branching ratios. Values for $\mathrm{Fe}(\mathrm{Cl})_{6}$ are from reference, ${ }^{79} \mathrm{Fe}(\mathrm{CN})_{6}$ from reference ${ }^{91}$ and $M n(a c a c)_{x}$ from reference. ${ }^{15}$ The yellow area indicate shows results within the maximum deviation from experiment (0.10). Only complexes with well-established electronic structures (blue dots) are included when calculating mean signed and maximum errors.

$\left[F e^{I I I}(P)(I m H)_{2}\right]^{+}$is a low-spin $d^{5}$ complex with a doublet $(S=1 / 2)$ ground state. On the low-energy side of the $L_{3}$ edge there is sharp peak, see Figure 3 , assigned to a $2 \mathrm{p} \rightarrow t_{2 g}$ channel. This is followed by the main $e_{g}$ peak, which is significantly broader compared to 
the $F e^{I I}(P)(I m H)_{2}$ spectrum. This is consistent with increased multiplet splittings when the unpaired $e_{g}$ electron interacts with the $t_{2 g}$ hole in the final state. There is no peak on the high-energy side, which can be explained by the very limited $\pi$ back-bonding. ${ }^{18,47}$

The RASPT2 simulations using the standard active space reproduces all experimental features, the presence of the $t_{2 g}$ peak, the multiplet broadening of the $e_{g}$ feature and the weaker intensity on the high-energy side. The differences are that the intensity of the $t_{2 g}$ peak is underestimated and the intensity drops off too fast at higher energies. The good agreement over a large part of the $L_{3}$ edge gives a CS of 0.990 , similar to the best results for the ferrous complex. For the ferric complex, the similarity increases with more states and RASPT2 gives better results than RASSCF, see SI Section 5.2.

It is clear from a visual inspection that the RAS simulations reproduce the changes in spectral shape associated with a change in oxidation state. To quantify this, the CS scores were calculated for the RAS spectra of a given complex and compared to the experimental spectra of the other complexes. These incorrect pairings gave similarity scores of 0.866 for the ferrous simulation applied to the ferric experiment, and 0.944 for the reverse combination, see Figure 6. These values are lower than those of the correct pairings, regardless of the method used for the simulation.

The experimental branching ratio of $\left[F e^{I I I}(P)(\operatorname{ImH})_{2}\right]^{+}$is 0.67 , slightly smaller than 0.68 measured for the ferrous complex. For this system, the RASPT2 calculations give a value of 0.63 , see Figure 5 . This is again an underestimation, but by a smaller amount than for the ferrous complex.

In addition to spectral shape and branching ratio, the redox state can also be reflected in the edge position. ${ }^{17,20}$ For $\mathrm{Fe}(\mathrm{P})(\mathrm{ImH})_{2}$ the maximum of the $L_{3}$ edge of the ferric complex is $1.0 \mathrm{eV}$ higher compared to the ferrous one, see Figure 3. Theoretical spectra are aligned to experiment and to correctly predict the oxidation-state shift the energy corrections should be the same for both complexes. This requires that the similar active spaces is used in both calculations. Furthermore, the shift depends on the number of final states in the RASSCF 


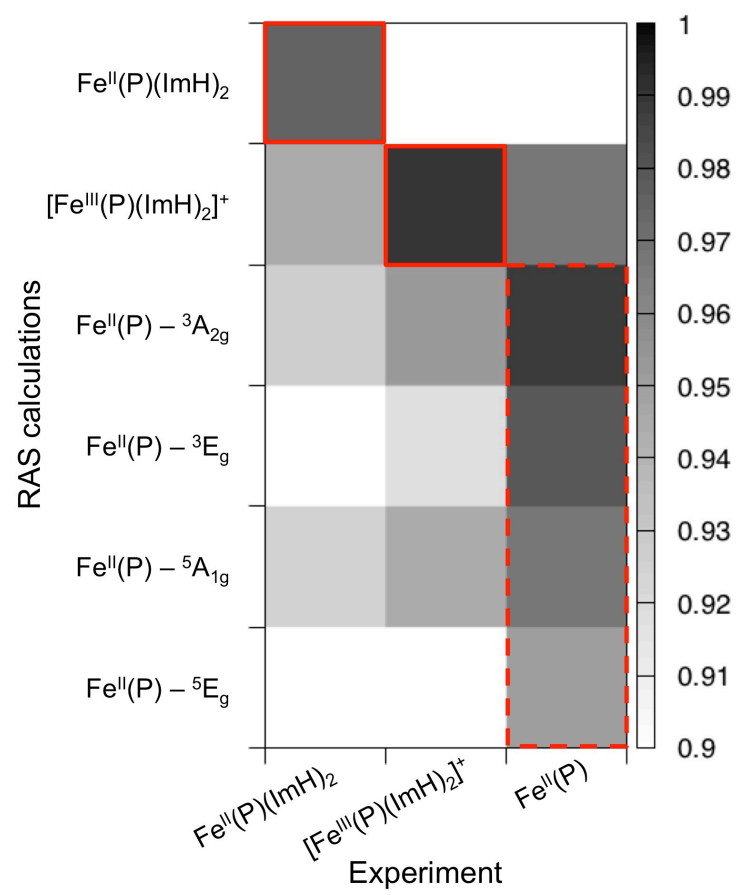

Figure 6: Cosine similarities for all possible combinations of three experimental and six simulated spectra. Solid red boxes show correct electronic structures and dashed red boxes indicate the correct complex. Numerical values are shown in Table SI 3.

step. Comparing calculations with equal number of states, the predicted energy shift varies between 0.96 and $1.79 \mathrm{eV}$, see Figure 7 .

Another variable sensitive to oxidation state is the integrated absorption intensity as this, at least partially, reflects the number of holes in metal-dominated orbitals. For $\mathrm{Fe}(\mathrm{P})(\mathrm{ImH})_{2}$ this intensity, normalized using an edge jump of 1, increases from 38 to 46, when going from the ferrous to the ferric complex. ${ }^{16,27}$ The increase of $21 \%$ is largely consistent with an increase in the number of holes from four to five. The calculated intensities are scaled to experiment and to predict intensity changes requires that these scaling factors are similar for two complexes. Again comparing calculations with equal number of states, the predicted intensity change varies between $22 \%$ and $27 \%$, see Figure 7 . 


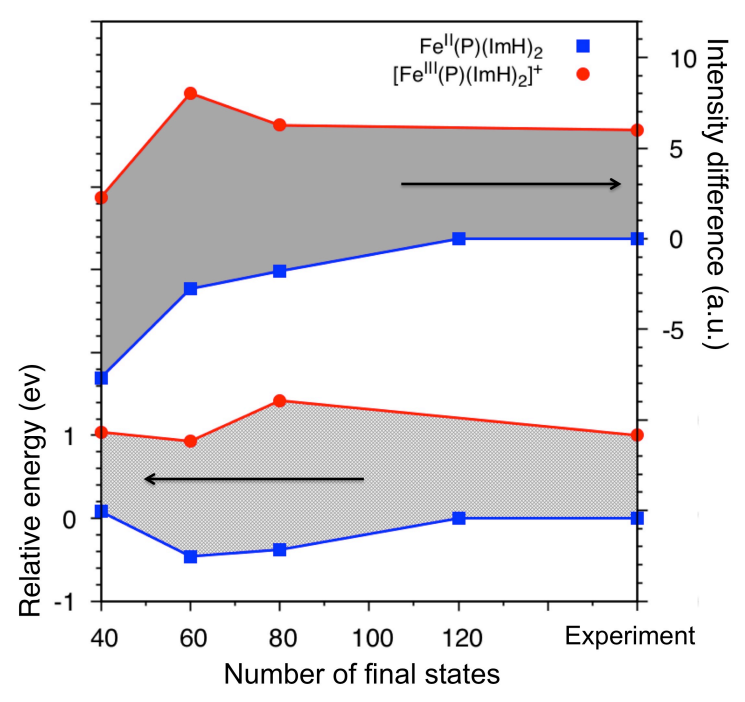

Figure 7: Energy difference of the maxima of the $L_{3}$ edges and integrated intensity difference for $\mathrm{Fe}^{I I}(\mathrm{P})(\mathrm{ImH})_{2}$ and $\left[\mathrm{Fe}^{I I I}(\mathrm{P})(\mathrm{ImH})_{2}\right]^{+}$from experiment and RAS calculations. Intensities are normalized using an edge jump of $1 .{ }^{18}$

\section{Fingerprints of electronic states}

For $F e^{I I}(P)$ the lack of axial ligands gives rise to several close-lying d-orbital levels. This leads to a large number of low-lying electronic states, differing in symmetry and spin multiplicity, see Figure 8. The key factor is the ordering of the metal $d_{x z}$ and $d_{y z}$ orbitals relative to other metal-dominated orbitals. ${ }^{54}$ These energies depend on the interactions with the $\pi$ system of the porphyrin, which rely on correlation pathways involving a large number of orbital interactions. ${ }^{71}$

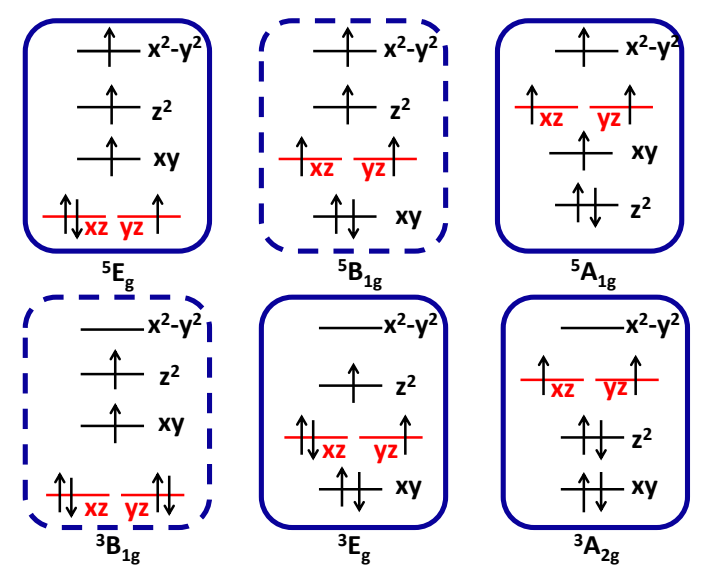

Figure 8: Schematic representations of selected low-lying states in $F e^{I I}(P)$. X-ray spectra have been simulated for the states within solid frames. 
The experimental $F e^{I I}(P)$ spectrum has a broad $L_{3}$ edge, with peaks at $705.7,706.5$ and a main peak at $708.1 \mathrm{eV}$, see Figure $9 .{ }^{27}$ There are shoulders both on the low- and high-energy side of $L_{3}$ edge. The broad edge reflects the large number of possible multiplet structures available with several unpaired electrons already in the ground state. The $L_{2}$ edge is also broad with a maximum at $\sim 720 \mathrm{eV}$. The branching ratio is 0.76 , which is larger than for the low-spin Fe(II) complex (ratio of 0.68), consistent with a higher spin multiplicity. ${ }^{22}$
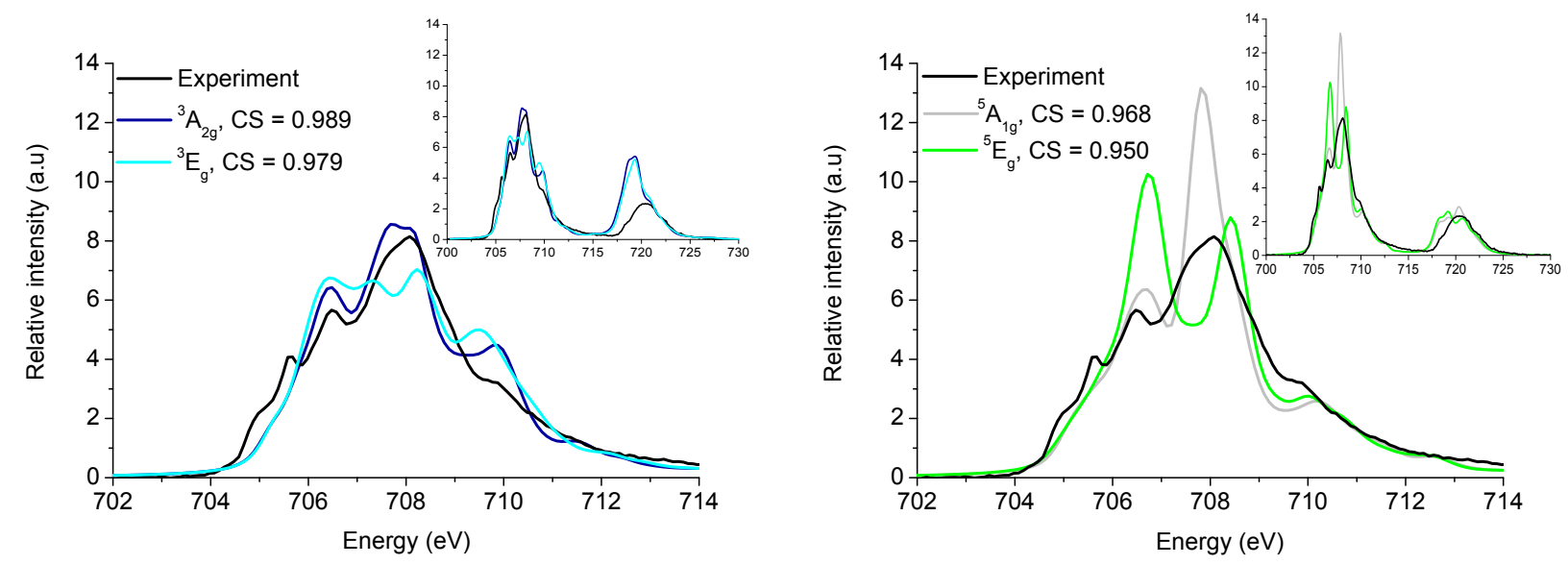

Figure 9: RASPT2/ANO-RCC-VDZP $L_{3}$ edge of $F e^{I I}(P)$ calculated using different lowlying electronic states with associated cosine similarities. Triplet states $(\mathrm{S}=1)$ to the left and quintet states $(\mathrm{S}=2)$ to the right, respectively. The full spectra comparisons are inserted in the right upper corners.

Four low-energy states as identified in previous high-level CASPT2 calculations have been simulated, ${ }^{3} A_{2 g},{ }^{3} E_{g},{ }^{5} A_{1 g}$, and ${ }^{5} E_{g} \cdot{ }^{63}$ The best agreement is obtained for the ${ }^{3} A_{2 g}$ simulation, which nicely reproduces most spectral features, see Figure 9. The similarity score is 0.989 , close to the values obtained for the $\left[\mathrm{Fe}(\mathrm{P})(\mathrm{ImH})_{2}\right]^{0 / 1+}$ complexes, see Figure 6. The main deviation is that the $L_{2}$ edge is too intense compared to experiment, see Figure 9. This leads to a branching ratio of 0.62 , well below the experimental value of 0.76 , see Figure 5. The ${ }^{3} E_{g}$ simulation gives a slightly lower match (0.979) and no improvement in the branching ratio. The ${ }^{5} A_{1 g}$ spectrum has two well-separated $L_{3}$ peaks, in contrast to the broader experimental spectrum, see Figure 9. This gives a lower CS score (0.971) compared to ${ }^{3} A_{2 g}$. However, the agreement with the experimental branching ratio $(0.73$ vs. 0.76$)$ is 
much better than for the triplets. Again, the ${ }^{5} E_{g}$ show some resemblance to the spectra with the same multiplicity, especially when it comes to the branching ratios, see Figures 5 and 9.

Comparing the spectra calculated at the same geometries, e.g., the triplet states at the optimized quintet geometry, changes the ordering of the ${ }^{3} A_{2 g}$ and ${ }^{3} E_{g}$ states, but the triplets show higher similarity than the quintets regardless of geometry. The similarity scores do not change when using the larger ANO-RCC-VTZP basis. There are also no major differences between RASSCF and RASPT2 or when changing the number of states. All these results are shown in SI Section 5.3. Other similarity measures, like the Euclidian distance, also gives the same ranking of the states, see SI Section 4.

The similarity analysis and branching ratios thus give different assignments of the groundstate spin multiplicity. This inconsistency could possibly be due to significant mixing of electronic states, as has already been invoked to explain the unusually large magnetic moments of $F e^{I I}(T P P)$ and $F e^{I I}(O E P) .{ }^{76,92,93}$ Taking a linear combination of the ${ }^{3} A_{2 g}$ spectrum, which has the best score, with each of other states further improves the CS score with maxima around $80-90 \%{ }^{3} A_{2 g}$, see Figure 10 (left). At the same time the branching ratio improves when mixing in quintet states, and when the fraction ${ }^{3} A_{2 g}$ is below $80 \%$ the branching ratio is within the largest error of any other complex, see Figure 10 (right). Similar results are obtained when using the same triplet geometry for all states, see Section SI 5.3.

A more stringent way to mix the states is to change their relative energies in the spin-orbit coupling scheme. For the mixing of ${ }^{3} A_{2 g}$ and ${ }^{5} A_{1 g}$ there is no maximum in the CS score, but the value does not decrease significantly until the energy difference goes down to $0.1 \mathrm{eV}$, see Figure 11. At the same time, the branching ratio improves significantly. For other states, the spin-orbit mixing procedure gives a maximum in the CS score but the improvement is relatively small, see SI Section 5.3. 

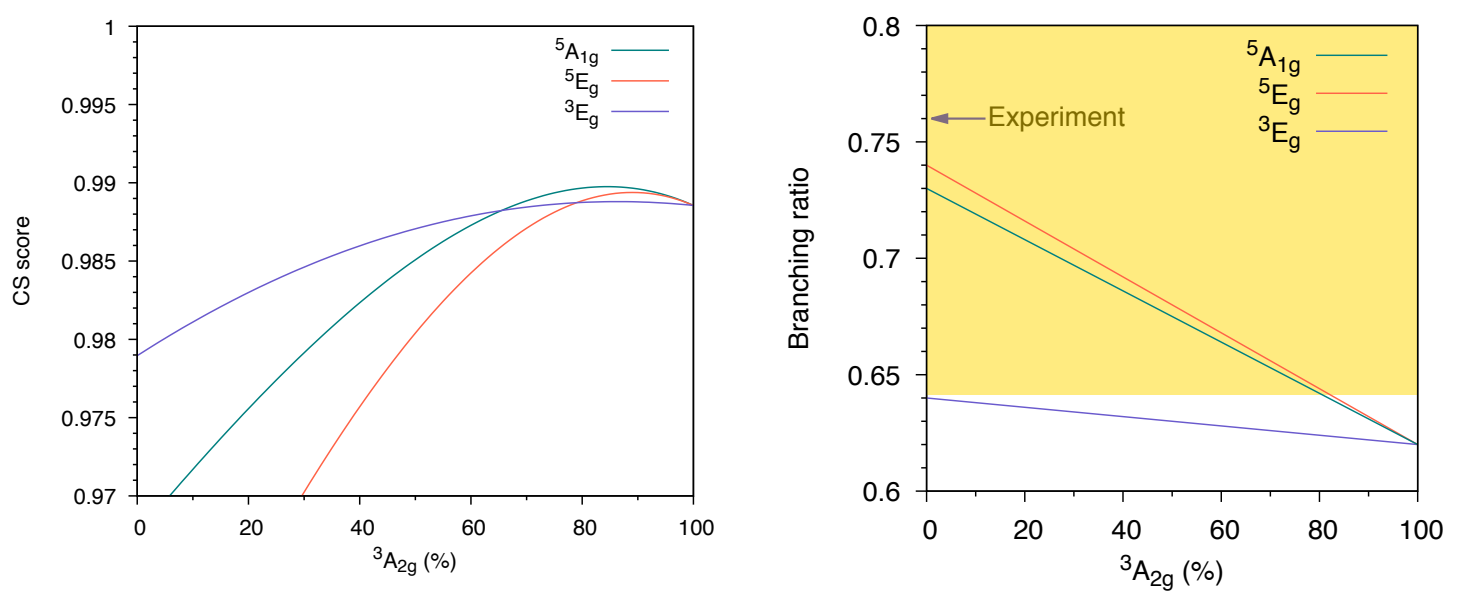

Figure 10: Cosine similarity score (left) and branching ratio (right) for mixing of the $F e^{I I}(P)$ ${ }^{3} A_{2 g}$ state with the ${ }^{5} A_{1 g},{ }^{3} E_{g}$, and ${ }^{5} E_{g}$ states. Results are given for a linear combination of the spectra of individual states, with triplets and quintets calculated in ${ }^{3} A_{2 g}$ geometry and ${ }^{5} A_{1 g}$ geometry respectively. The yellow band shows results within the maximum deviation as estimated in Figure 5.

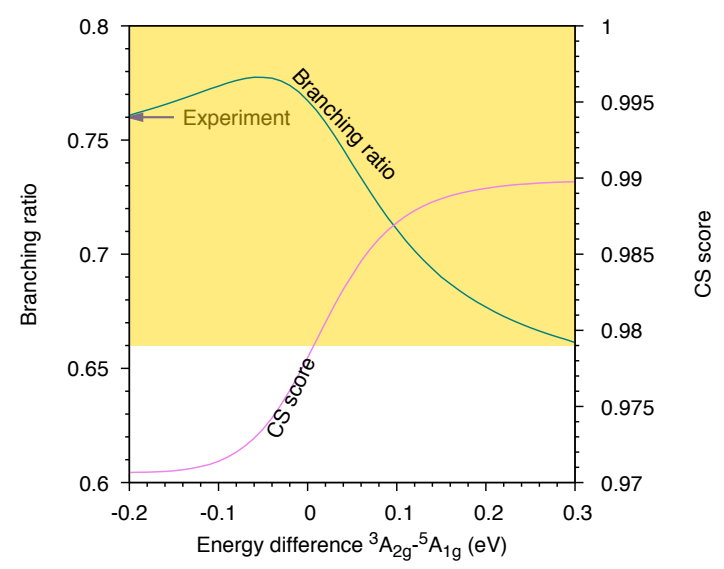

Figure 11: Cosine similarity score and branching ratio for mixing of the $F e^{I I}(P){ }^{3} A_{2 g}$ and ${ }^{5} A_{1 g}$ states by varying their relative energy. Calculations are done at the ${ }^{3} A_{2 g}$ geometry. The yellow band shows results within the maximum deviation of the branching ratio as estimated in Figure 5. 


\section{Discussion}

As mentioned above, the three studied complexes together approximate the limiting descriptions of the Fe- $\mathrm{O}_{2}$ bond in hemoglobin. Oxygen binding to $\mathrm{Fe}(\mathrm{II})$ results in a singlet, which is inaccessible for many standard probes. This singlet can be formed from a combination of different spin multiplicities of iron and oxygen, with antiparallel alignment of either two singlets $\mathrm{Fe}^{I I}-\mathrm{O}_{2}$, two doublets $\mathrm{Fe} e^{I I I}-\mathrm{O}_{2}^{-}$or two triplets $\mathrm{Fe}^{I I}-\mathrm{O}_{2}$. The possibility to distinguish between these alternative electronic structures is here analyzed for four observables, spectral shape, branching ratio, incident energy, and integrated absorption intensity.

Starting with spectral shape, the use of the cosine similarity makes it possible to quantify the accuracy and compare between different systems. The two $\left[\mathrm{Fe}(\mathrm{P})(\mathrm{ImH})_{2}\right]^{0 / 1+}$ complexes with well-established ground states illustrate the expected accuracy of the RASPT2 simulations. All major spectral features are included, although with errors in relative intensity and energy similar to what has previously been observed. ${ }^{79}$ The highest similarity scores were 0.99 , which can be used as a guideline for what can be achieved with a correct application of the RASPT2 method. However, for $F e^{I I}(P)(\operatorname{ImH})_{2}$ the high similarity was reached only by modifying the active space. The increase in intensity of the low-energy region when adding filled orbitals matches the observations from CTM calculations. ${ }^{18}$ However, due to the restrictions in the excitation space the modified active spaces are not of higher quality than the standard active space. Placing the $\sigma$ bonding and antibonding orbitals in different subspaces in RASPT2 ignores the non-diagonal Fock matrix elements between them, which could be argued to deteriorate the results. ${ }^{37}$ The low-energy transitions appear in all alternative active spaces, also when using the same number of orbitals as the standard active space, but only in RASPT2 and not in RASSCF. It is thus possible that the higher similarity score is simply due to effects of an incomplete PT2 treatment. These results show the importance of the choice of active space for the simulations. This makes RAS less predictive, as it can be difficult to determine whether a deviation from experiment is due to an insufficient active space or an incorrect electronic state. 
To understand how well the RAS simulations can distinguish between the three alternative electronic structures, all experimental spectra were matched against all six calculated spectra, see Figure 6. Based on the CS scores, the simulations identify the correct electronic state directly from the spectral shape. The smallest difference between a correct and an incorrect spectrum is 0.02 , with the incorrect one being $\left[\mathrm{Fe}^{I I I}(\mathrm{P})(\mathrm{ImH})_{2}\right]^{+}$applied to $F e^{I I}(P)$. To get statistically reliable results requires a much larger benchmark set. Assuming that the difference is 0.02 between correct and incorrect spectra, effects on the CS score larger than 0.01 should be considered significant.

Inclusion of dynamical correlation has previously led to significant improvements, especially for systems with strong ligand fields. ${ }^{79,91}$ For $\left[\mathrm{Fe}^{I I I}(\mathrm{P})(\mathrm{ImH})_{2}\right]^{1+}$ the CS score increases from 0.976 to 0.990 when going from RASSCF to RASPT2 due to an improved description of the $t_{2 g^{-}} e_{g}$ ligand-field splitting, see SI Section 5.2. For the other two systems there are no significant improvements with PT2, possibly because the spectral shapes are dominated by the multiplet splittings that are well described inside the active space. The results further depend on the number of final states included in the calculations. For the present systems, the CS score varies by less than 0.01 , but large changes have been observed previously. ${ }^{79}$ More final states do not consistently give better matches, see SI Sections 5.15.3 , which makes it difficult to give a definitive recommendation. Finally, increasing the size of the basis set from ANO-RCC-VDZP to ANO-RCC-VTZP in the $F e^{I I}(P)$ calculation changed CS scores by less than 0.005. Numerical evaluation of the effects of different model choices makes it possible to optimize the balance between accuracy and computational cost. A large active space with a smaller basis set is recommended, and PT2 corrections should be added if computationally feasible.

When it comes to distinguishing between the close-lying electronic states of $F e^{I I}(P)$, the similarity analysis favors the ${ }^{3} A_{2 g}$ state among the four tested alternatives although the difference to ${ }^{3} E_{g}$ is small. $S=1$ is the spin state of most four-coordinate $F e^{I I}(P)$ systems, but is not consistent with the original $S=2$ assignment for solid $F e^{I I}(T p i v P P) .{ }^{73}$ Con- 
sidering the delicate balance between spin states in $F e^{I I}(T p i v P P)$, a possible explanation for this discrepancy could be differences in sample preparation. As the X-ray spectra are collected at temperatures around $220 \mathrm{~K},{ }^{27}$ while other characterizations were made at room temperature, another possibility is that the system undergoes a temperature-driven change in spin state, where the triplet gets more stable with decreasing temperature.

The branching ratio is an alternative tool to assign electronic structure as it is sensitive to the spin state. Comparisons of the results from seven complexes shows that the RAS simulations underestimate the branching ratio in this small sample with a mean signed deviation of -0.014, see Figure 5. Unfortunately, for some complexes there are rather large deviations between calculation and experiment, with a maximum absolute deviation of 0.10 , or $15 \%$. One possible reason could be experimental uncertainties as the ratio is sensitive to the modeling of the edge jump as well as differences in decay processes between the edges. Still, it can be established with reasonable accuracy in transmission and total electron yield collection modes. A large part of the deviation is therefore likely to come from errors in the calculations. For some systems, the branching ratio is sensitive to the number of final states and the deviations could come from the use of a perturbative treatment of the strong spin-orbit coupling with an incomplete set of states. A more accurate treatment would be to include the spin-orbit coupling in the orbital optimization, either in a 2- or 4-component approach. ${ }^{28,32,94,95}$

Changing the oxidation state only results in a change in the branching ratio by 0.02 , which is small compared to the expected deviatons. However, the calculations predict large effects on the branching ratio for different spin states of $F e^{I I}(P)$, consistent with observed differences between high- and low-spin iron complexes. ${ }^{96}$ Just looking at the branching ratios, quintet states are much better matches than triplets, see Figure 5. The simulated triplet spectra significantly underestimate the branching ratio, but the agreeement improves by mixing in quintet contributions through spin-orbit coupling. Unfortunately, the large uncertainties prevent a definitive assignment of spin state from the branching ratio. When 
it comes to electronic structures with the same multiplicity, both branching ratios and similarity scores are close. Energy calculations will often be more appropriate such states, and the two approaches can always be used to complement each other.

Although spectral shape is a powerful probe of oxidation state, it can sometimes be easier to identify a change in oxidation state through the spectral shift, e.g., in the presence of more than one overlapping species. For $\left[\mathrm{Fe}(\mathrm{P})(\mathrm{ImH})_{2}\right]^{0 / 1+}$ the shift is $1.0 \mathrm{eV}$. RAS gives a shift in the right direction, but the value depends on the number of final states and errors are up to $0.8 \mathrm{eV}$. This can be compared to an error of $0.3 \mathrm{eV}$ for the $2.0-\mathrm{eV}$ shift observed for two high-spin manganese complexes where the active space was so small that all possible final states could be included. ${ }^{21}$ The dependence of the shift on the number of states can be overcome using response theory, but this is not implemented at the PT2 level. The integrated absorption intensity can also be used to separate species of different oxidation states, and here the changes between ferrous and ferric systems are reasonably well described.

A limitation in the present analysis is that the simulation can deviate from experiment both in intensity and the relative energy of different features. None of the tested similarity measures account for peaks that are shifted in energy more than the spectral broadening. For the heme systems, most features are predicted with only small energy differences, with the exception of the position of the $L_{2}$ edge relative to the $L_{3}$ edge. As long as the analysis is performed only over one edge at the time, the CS score gives reasonable results for the present systems. Although the current analysis has been performed for L-edge XAS, the same approach can also be applied to other types of X-ray spectroscopies e.g., XAS and RIXS in the metal K pre edge for which we have recently extended the RASPT2 approach. ${ }^{91,97,98}$

\section{Conclusions}

The analysis shows how spin state and symmetry can be assigned from multiconfigurational simulations of X-ray spectra as an alternative to more and more sophisticated energy calcula- 
tions. RASPT2 calculations reproduce essentially all features of the metal L-edge XAS of the three heme systems. This shows that good accuracy can be achieved also for medium-sized molecules using moderate active spaces and small basis sets. By introducing a numerical estimate of the similarity between experiment and calculation, it is shown that spectral shape can distinguish between alternative electronic structures. Significant errors in the calculated branching ratios make them a less reliable variable. Integrated absorption intensity and incident energy shift can be used to identify oxidation states. The former is well described with marginal errors in total intensity when comparing the two $\left[\mathrm{Fe}(\mathrm{P})(\mathrm{ImH})_{2}\right]^{0 / 1+}$ complexes. However, the calculations of the incident energy shift between the two complexes depend on the number of final states included in the calculations. For solid $F e^{I I}(T p i v P P)$, the spectral shape indicates a triplet state. However, to reach decent agreement also for the branching ratio requires contributions of quintet states through spin-orbit coupling. It is possible that the system undergoes a temperature-dependent spin transition from $S=2$ at room temperature to $S=1$ at $220 \mathrm{~K}$. The three complexes tested here represent limiting electronic structures for $\mathrm{O}_{2}$ binding to heme and RAS simulation of X-ray spectra should be able to separate these three alternatives. This shows that RAS is a promising method to identify different electronic structures during catalytic reactions from their X-ray fingerprint.

\section{Acknowledgement}

We acknowledge financial support from the Swedish Research Council (M.L. grant 2012-3924 and R.L. grant 2016-03398), the Carl Trygger Foundation, the Petra och Karl Erik Hedborgs Foundation, and the Knut and Alice Wallenberg Foundation (Grant No. KAW-2013.0020). The computations were performed on resources provided by SNIC trough Uppsala Multidisciplinary Center for Advanced Computational Science (UPPMAX) under project snic2016-

1-464 and National Supercomputer Centre at Linköping University (Triolith) under projects snic2014-5-36, and snic2016-1-508. 


\section{Supporting Information Available}

The Supporting Information includes additional computational details and results, as well as Cartesian coordinates for all structures. This material is available free of charge via the Internet at http://pubs . acs .org/.

\section{References}

(1) Gonzalez-Jimenez, I. D.; Cats, K.; Davidian, T.; Ruitenbeek, M.; Meirer, F.; Liu, Y.; Nelson, J.; Andrews, J. C.; Pianetta, P.; de Groot, F. M. Hard X-ray Nanotomography of Catalytic Solids at Work. Angew. Chem. Int. Ed. 2012, 51, 11986-11990.

(2) Seefeldt, L. C.; Hoffman, B. M.; Dean, D. R. Electron transfer in nitrogenase catalysis. Curr. Opin. Chem. Biol. 2012, 16, 19-25.

(3) Yano, J.; Yachandra, V. Mn4Ca cluster in photosynthesis: where and how water is oxidized to dioxygen. Chem. Rev. 2014, 114, 4175-4205.

(4) Wernet, P.; Kunnus, K.; Josefsson, I.; Rajkovic, I.; Quevedo, W.; Beye, M.; Schreck, S.; Gruebel, S.; Scholz, M.; Nordlund, D.; Zhang, W.; Hartsock, R. W.; Schlotter, W. F.; Turner, J. J.; Kennedy, B.; Hennies, F.; de Groot, F. M. F.; Gaffney, K. J.; Techert, S.; Odelius, M.; Foehlisch, A. Orbital-specific mapping of the ligand exchange dynamics of $\mathrm{Fe}(\mathrm{CO})_{5}$ in solution. Nature 2015, 520, 78-81.

(5) Hugenbruch, S.; Shafaat, H. S.; Krämer, T.; Delgado-Jaime, M. U.; Weber, K.; Neese, F.; Lubitz, W.; DeBeer, S. In search of metal hydrides: an X-ray absorption and emission study of [NiFe] hydrogenase model complexes. Phys. Chem. Chem. Phys. 2016, 18, 10688-10699.

(6) Gorlin, Y.; Chung, C.-J.; Nordlund, D.; Clemens, B. M.; Jaramillo, T. F. Mn3O4 sup- 
ported on glassy carbon: an active non-precious metal catalyst for the oxygen reduction reaction. ACS. Catal. 2012, 2, 2687-2694.

(7) Kern, J.; Alonso-Mori, R.; Tran, R.; Hattne, J.; Gildea, R. J.; Echols, N.; Glöckner, C.; Hellmich, J.; Laksmono, H.; Sierra, R. G. Simultaneous femtosecond X-ray spectroscopy and diffraction of photosystem II at room temperature. Science 2013, 340, 491-495.

(8) Ebrahimizadeh Abrishami, M.; Risch, M.; Scholz, J.; Roddatis, V.; Osterthun, N.; Jooss, C. Oxygen Evolution at Manganite Perovskite Ruddlesden-Popper Type Particles: Trends of Activity on Structure, Valence and Covalence. Materials 2016, 9, 921.

(9) Kowalska, J. K.; Nayyar, B.; Rees, J. A.; Schiewer, C. E.; Lee, S. C.; Kovacs, J. A.; Meyer, F.; Weyherméżóler, T.; Otero, E.; DeBeer, S. Iron L 2,3 -Edge X-ray Absorption and X-ray Magnetic Circular Dichroism Studies of Molecular Iron Complexes with Relevance to the FeMoco and FeVco Active Sites of Nitrogenase. Inorg. Chem. 2017, $56,8147-8158$.

(10) Mara, M. W.; Hadt, R. G.; Reinhard, M. E.; Kroll, T.; Lim, H.; Hartsock, R. W.; Alonso-Mori, R.; Chollet, M.; Glownia, J. M.; Nelson, S. Metalloprotein entatic control of ligand-metal bonds quantified by ultrafast x-ray spectroscopy. Science 2017, 356, $1276-1280$.

(11) Schreck, S.; Gavrila, G.; Weniger, C.; Wernet, P. A sample holder for soft x-ray absorption spectroscopy of liquids in transmission mode. Rev. Sci. Instrum. 2011, 82, 103101.

(12) Mitzner, R.; Rehanek, J.; Kern, J.; Gul, S.; Hattne, J.; Taguchi, T.; Alonso-Mori, R.; Tran, R.; Weniger, C.; Schröder, H. L-edge X-ray absorption spectroscopy of dilute systems relevant to metalloproteins using an X-ray free-electron laser. J. Phys. Chem. Lett. 2013, 4, 3641-3647. 
(13) Ekimova, M.; Quevedo, W.; Faubel, M.; Wernet, P.; Nibbering, E. T. A liquid flatjet system for solution phase soft-x-ray spectroscopy. Struct. Dynam. 2015, 2, 054301.

(14) Kubin, M.; Kern, J.; Gul, S.; Kroll, T.; Chatterjee, R.; Löchel, H.; Fuller, F. D.; Sierra, R. G.; Quevedo, W.; Weniger, C. Soft x-ray absorption spectroscopy of metalloproteins and high-valent metal-complexes at room temperature using free-electron lasers. Struct. Dynam. 2017, 4, 054307.

(15) Kubin, M.; Guo, M.; Ekimova, M.; Baker, M. L.; Kroll, T.; Källman, E.; Kern, J.; Yachandra, V. K.; Yano, J.; Nibbering, E. T.; Lundberg, M.; Wernet, P. Direct Determination of Absolute Absorption Cross Sections at the L-Edge of Dilute Mn Complexes in Solution Using a Transmission Flatjet. Inorg. Chem. 2018, 57, 5449-5462.

(16) Wasinger, E. C.; De Groot, F. M.; Hedman, B.; Hodgson, K. O.; Solomon, E. I. L-edge X-ray absorption spectroscopy of non-heme iron sites: experimental determination of differential orbital covalency. J. Am. Chem. Soc. 2003, 125, 12894-12906.

(17) Hocking, R. K.; Wasinger, E. C.; de Groot, F. M.; Hodgson, K. O.; Hedman, B.; Solomon, E. I. Fe L-edge XAS studies of $\mathrm{K}_{4}\left[\mathrm{Fe}(\mathrm{CN})_{6}\right]$ and $\mathrm{K}_{3}\left[\mathrm{Fe}(\mathrm{CN})_{6}\right]$ : A direct probe of back-bonding. J. Am. Chem. Soc. 2006, 128, 10442-10451.

(18) Hocking, R. K.; Wasinger, E. C.; Yan, Y.-L.; de Groot, F. M.; Walker, F. A.; Hodgson, K. O.; Hedman, B.; Solomon, E. I. Fe L-edge X-ray absorption spectroscopy of low-spin heme relative to non-heme Fe complexes: Delocalization of Fe d-electrons into the porphyrin ligand. J. Am. Chem. Soc. 2007, 129, 113-125.

(19) Kroll, T.; Hadt, R. G.; Wilson, S. A.; Lundberg, M.; Yan, J. J.; Weng, T.-C.; Sokaras, D.; Alonso-Mori, R.; Casa, D.; Upton, M. H.; Hedman, B.; Hodgson, K. O.; Solomon, E. I. Resonant Inelastic X-ray Scattering on Ferrous and Ferric Bis-imidazole Porphyrin and Cytochrome c: Nature and Role of the Axial Methionine-Fe Bond. J. Am. Chem. Soc. 2014, 136, 18087-18099. 
(20) Cramer, S.; de Groot, F.; Ma, Y.; Chen, C.; Sette, F.; Kipke, C.; Eichhorn, D.; Chan, M.; Armstrong, W. Ligand field strengths and oxidation states from manganese L-edge spectroscopy. J. Am. Chem. Soc. 1991, 113, 7937-7940.

(21) Kubin, M.; Guo, M.; Kroll, T.; Löchel, H.; Källman, E.; Baker, M. L.; Mitzner, R.; Gul, S.; Kern, J.; Föhlisch, A.; Erko, A.; Bergmann, U.; Yachandra, V. K.; Yano, J.; Lundberg, M.; Wernet, P. Probing the Oxidation State of Transition Metal Complexes: A Case Study on How Charge and Spin Densities Determine Mn L-Edge X-ray Absorption Energies. Chem. Sci. 2018, 9, 6813-6829.

(22) Thole, B.; Van der Laan, G. Branching ratio in x-ray absorption spectroscopy. Phys. Rev. B 1988, 38, 3158 .

(23) Pierloot, K.; Eftimios, T. 3pd intershell correlation effects in transition metal ions. Chem. Phys. Lett. 1993, 214, 583-590.

(24) Vancoillie, S.; Zhao, H.; Tran, V. T.; Hendrickx, M. F.; Pierloot, K. Multiconfigurational second-order perturbation theory restricted active space (RASPT2) studies on mononuclear first-row transition-metal systems. J. Chem. Theory. Comput. 2011, 7 , $3961-3977$.

(25) Tanaka, A.; Jo, T. Resonant 3d, 3p and 3s photoemission in transition metal oxides predicted at 2p threshold. J. Phys. Soc. JPN 1994, 63, 2788-2807.

(26) de Groot, F. Multiplet effects in X-ray spectroscopy. Coordin. Chem. Rev. 2005, 249, $31-63$.

(27) Wilson, S. A.; Kroll, T.; Decreau, R. A.; Hocking, R. K.; Lundberg, M.; Hedman, B.; Hodgson, K. O.; Solomon, E. I. Iron L-Edge X-ray absorption spectroscopy of oxypicket fence porphyrin: experimental insight into $\mathrm{Fe}-\mathrm{O}_{2}$ bonding. J. Am. Chem. Soc. 2013, 135, 1124-1136. 
(28) Fronzoni, G.; Stener, M.; Decleva, P.; Wang, F.; Ziegler, T.; Van Lenthe, E.; Baerends, E. Spin-orbit relativistic time dependent density functional theory calculations for the description of core electron excitations: $\mathrm{TiCl}_{4}$ case study. Chem. Phys. Lett. 2005, 416, 56-63.

(29) Bunău, O.; Joly, Y. Full potential x-ray absorption calculations using time dependent density functional theory. J. Phys. Condens. Mat. 2012, 24, 215502.

(30) Josefsson, I.; Kunnus, K.; Schreck, S.; Föhlisch, A.; de Groot, F.; Wernet, P.; Odelius, M. Ab Initio Calculations of X-ray Spectra: Atomic Multiplet and Molecular Orbital Effects in a Multiconfigurational SCF Approach to the L-Edge Spectra of Transition Metal Complexes. J. Phys. Chem. Lett. 2012, 3, 3565-3570.

(31) Roemelt, M.; Maganas, D.; DeBeer, S.; Neese, F. A combined DFT and restricted open-shell configuration interaction method including spin-orbit coupling: Application to transition metal L-edge X-ray absorption spectroscopy. J. Chem. Phys. 2013, 138, 204101.

(32) Bagus, P. S.; Nelin, C. J.; Ilton, E. S.; Sassi, M. J.; Rosso, K. M. Analysis of X-ray adsorption edges: $\mathrm{L}_{2,3}$ edge of $\mathrm{FeCl}_{4}^{-}$. J. Chem. Phys. 2017, 147, 224306.

(33) Kasper, J. M.; Lestrange, P. J.; Stetina, T. F.; Li, X. Modeling L 2,3 -Edge X-ray Absorption Spectroscopy with Real-Time Exact Two-Component Relativistic Time-Dependent Density Functional Theory. J. Chem. Theory Comput. 2018, 14, 1998-2006, PMID: 29561613.

(34) Chantzis, A.; Kowalska, J. K.; Maganas, D.; DeBeer, S.; Neese, F. Ab initio Wavefunction-based Determination of Element Specific Shifts for the Efficient Calculation of X-Ray Absorption Spectra of Main Group Elements and First Row Transition Metals. J. Chem. Theory Comput. 2018, 14, 3686-3702. 
(35) Olsen, J.; Roos, B. O.; Jørgensen, P.; Jensen, H. J. Å. Determinant based configuration interaction algorithms for complete and restricted configuration interaction spaces. $J$. Chem. Phys. 1988, 89, 2185-2192.

(36) Malmqvist, P. A.; Rendell, A.; Roos, B. O. The restricted active space self-consistentfield method, implemented with a split graph unitary group approach. J. Phys. Chem. 1990, $94,5477-5482$.

(37) Malmqvist, P.-Å.; Pierloot, K.; Shahi, A. R. M.; Cramer, C. J.; Gagliardi, L. The restricted active space followed by second-order perturbation theory method: Theory and application to the study of $\mathrm{CuO}_{2}$ and $\mathrm{Cu}_{2} \mathrm{O}_{2}$ systems. J. Chem. Phys. 2008, 128, 204109.

(38) Wernet, P.; Kunnus, K.; Schreck, S.; Quevedo, W.; Kurian, R.; Techert, S.; de Groot, F. M.; Odelius, M.; Föhlisch, A. Dissecting Local Atomic and Intermolecular Interactions of Transition-Metal Ions in Solution with Selective X-ray Spectroscopy. J. Phys. Chem. Lett. 2012, 3, 3448-3453.

(39) Bokarev, S. I.; Dantz, M.; Suljoti, E.; Kühn, O.; Aziz, E. F. State-Dependent Electron Delocalization Dynamics at the Solute-Solvent Interface: Soft-X-Ray Absorption Spectroscopy and Ab Initio Calculations. Phys. Rev. Lett. 2013, 111, 083002-083007.

(40) Engel, N.; Bokarev, S. I.; Suljoti, E.; Garcia-Diez, R.; Lange, K. M.; Atak, K.; Golnak, R.; Kothe, A.; Dantz, M.; Kühn, O.; Aziz, E. F. Chemical bonding in aqueous ferrocyanide: experimental and theoretical X-ray spectroscopic study. J. Phys. Chem. B 2014, 118, 1555-1563.

(41) Pinjari, R. V.; Delcey, M. G.; Guo, M.; Odelius, M.; Lundberg, M. Restricted active space calculations of L-edge X-ray absorption spectra: From molecular orbitals to multiplet states. J. Chem. Phys. 2014, 141, 124116. 
(42) Pinjari, R. V.; Delcey, M. G.; Guo, M.; Odelius, M.; Lundberg, M. Erratum:Restricted active space calculations of L-edge X-ray absorption spectra: From molecular orbitals to multiplet states. J. Chem. Phys. 141, 124116 (2014)]. J. Chem. Phys. 2015, 142, 069901.

(43) Kunnus, K.; Zhang, W.; Delcey, M. G.; Pinjari, R. V.; Miedema, P. S.; Schreck, S.; Quevedo, W.; Schroeder, H.; Föhlisch, A.; Gaffney, K. J.; Lundberg, M.; Odelius, M.; Wernet, P. Viewing the valence electronic structure of ferric and ferrous hexacyanide in solution from the Fe and cyanide perspectives. J. Phys. Chem. B 2016, 120, 7182-7194.

(44) Debie, E.; De Gussem, E.; Dukor, R. K.; Herrebout, W.; Nafie, L. A.; Bultinck, P. A confidence level algorithm for the determination of absolute configuration using vibrational circular dichroism or Raman optical activity. ChemPhysChem 2011, 12, 1542-1549.

(45) Bruhn, T.; Schaumloeffel, A.; Hemberger, Y.; Bringmann, G. SpecDis: Quantifying the comparison of calculated and experimental electronic circular dichroism spectra. Chirality 2013, 25, 243-249.

(46) Lever, A. B. P.; Gray, H. B. In Iron Porphyrins; Addison-Wesley: Reading, MA, 1983.

(47) Kadish, K. M.; Smith, K. M.; Guilard, R. The Porphyrin Handbook: Inorganic, organometallic and coordination chemistry; Academic Press: New York, 2000; Vol. 3.

(48) Aziz, E. F.; Ottosson, N.; Bonhommeau, S.; Bergmann, N.; Eberhardt, W.; Chergui, M. Probing the electronic structure of the hemoglobin active center in physiological solutions. Phys. Rev. Lett. 2009, 102, 068103.

(49) Lange, K. M.; Golnak, R.; Bonhommeau, S.; Aziz, E. F. Ligand discrimination of myoglobin in solution: an iron L-edge X-ray absorption study of the active centre. Chem. Commun. 2013, 49, 4163-4165. 
(50) Wende, H.; Bernien, M.; Luo, J.; Sorg, C.; Ponpandian, N.; Kurde, J.; Miguel, J.; Piantek, M.; Xu, X.; Eckhold, P.; Kuch, W.; Baberschke, K.; Panchmatia, P. M.; Sanyal, B.; Oppeneer, P. M.; Eriksson, O. Substrate-induced magnetic ordering and switching of iron porphyrin molecules. Nat. Mater. 2007, 6, 516.

(51) Bhandary, S.; Brena, B.; Panchmatia, P. M.; Brumboiu, I.; Bernien, M.; Weis, C.; Krumme, B.; Etz, C.; Kuch, W.; Wende, H.; ; Eriksson, O.; Sanyal, B. Manipulation of spin state of iron porphyrin by chemisorption on magnetic substrates. Phys. Rev. B 2013, 88, 024401.

(52) Preuße, M.; Bokarev, S. I.; Aziz, S. G.; Kühn, O. Towards an ab initio theory for metal L-edge soft X-ray spectroscopy of molecular aggregates. Struct. Dynam. 2016, 3, 062601.

(53) Collman, J. P.; Hoard, J.; Kim, N.; Lang, G.; Reed, C. A. Synthesis, stereochemistry, and structure-related properties of $\alpha, \beta, \gamma, \delta$-tetraphenylporphinatoiron(II). J. Am. Chem. Soc. 1975, 97, 2676-2681.

(54) Goff, H.; La Mar, G. N.; Reed, C. A. Nuclear magnetic resonance investigation of magnetic and electronic properties of "intermediate spin" ferrous porphyrin complexes. J. Am. Chem. Soc. 1977, 99, 3641-3646.

(55) Kitagawa, T.; Teraoka, J. The resonance Raman spectra of intermediate-spin ferrous porphyrin. Chem. Phys. Lett. 1979, 63, 443-446.

(56) Obara, S.; Kashiwagi, H. Ab initio MO studies of electronic states and Mössbauer spectra of high-, intermediate-, and low-spin Fe(II)-porphyrin complexes. J. Chem. Phys. 1982, 77, 3155-3165.

(57) Choe, Y. K.; Nakajima, T.; Hirao, K.; Lindh, R. Theoretical study of the electronic ground state of iron(II) porphine. II. J. Chem. Phys. 1999, 111, 3837-3845. 
(58) Scherlis, D. A.; Estrin, D. A. Structure and spin-state energetics of an iron porphyrin model: An assessment of theoretical methods. Int. J. Quantum. Chem. 2002, 87, 158166.

(59) Pierloot, K. The CASPT2 method in inorganic electronic spectroscopy: from ionic transition metal to covalent actinide complexes. Mol. Phys. 2003, 101, 2083-2094.

(60) Groenhof, A. R.; Swart, M.; Ehlers, A. W.; Lammertsma, K. Electronic ground states of iron porphyrin and of the first species in the catalytic reaction cycle of cytochrome P450s. J. Phys. Chem. A 2005, 109, 3411-3417.

(61) Liao, M.-S.; Watts, J. D.; Huang, M.-J. Assessment of the performance of densityfunctional methods for calculations on iron porphyrins and related compounds. J. Comput. Chem. 2006, 27, 1577-1592.

(62) Ali, M. E.; Sanyal, B.; Oppeneer, P. M. Electronic structure, spin-states, and spincrossover reaction of heme-related Fe-porphyrins: a theoretical perspective. J. Phys. Chem. B 2012, 116, 5849-5859.

(63) Radon, M. Revisiting the role of exact exchange in DFT spin-state energetics of transition metal complexes. Phys. Chem. Chem. Phys. 2014, 16, 14479-14488.

(64) Radoń, M. Spin-state energetics of heme-related models from DFT and coupled cluster calculations. J. Chem. Theory. Comput. 2014, 10, 2306-2321.

(65) Zhao, H.; Fang, C.; Gao, J.; Liu, C. Spin-state energies of heme-related models from spin-flip TDDFT calculations. Phys. Chem. Chem. Phys. 2016, 18, 29486-29494.

(66) Phung, Q. M.; Wouters, S.; Pierloot, K. Cumulant Approximated Second-Order Perturbation Theory Based on the Density Matrix Renormalization Group for Transition Metal Complexes: A Benchmark Study. J. Chem. Theory. Comput. 2016, 12, 43524361. 
(67) Li Manni, G.; Smart, S. D.; Alavi, A. Combining the complete active space selfconsistent field method and the full configuration interaction quantum Monte Carlo within a super-CI framework, with application to challenging metal-porphyrins. $J$. Chem. Theory Comput. 2016, 12, 1245-1258.

(68) Pierloot, K.; Phung, Q. M.; Domingo, A. Spin State Energetics in First-Row Transition Metal Complexes: Contribution of (3s3p) Correlation and Its Description by SecondOrder Perturbation Theory. J. Chem. Theory. Comput. 2017, 13, 537-553.

(69) Smith, J. E.; Mussard, B.; Holmes, A. A.; Sharma, S. Cheap and near exact CASSCF with large active spaces. J. Chem. Theory Comput. 2017, 13, 5468-5478.

(70) Phung, Q. M.; Feldt, M.; Harvey, J. N.; Pierloot, K. Toward Highly Accurate Spin State Energetics in First-Row Transition Metal Complexes: A Combined CASPT2/CC Approach. J. Chem. Theory. Comput. 2018, 14, 2446-2455.

(71) Li Manni, G.; Alavi, A. Understanding the mechanism stabilizing intermediate spin states in Fe(II)-Porphyrin. J. Phys. Chem. A 2018, 122, 4935-4987.

(72) Collins, D.; Countryman, R.; Hoard, J. Stereochemistry of low-spin iron porphyrins. I. Bis(imidazole) $\alpha, \beta, \gamma, \delta$-tetraphenylporphinatoiron(III)chloride. J. Am. Chem. Soc. 1972, 94, 2066-2072.

(73) Collman, J. P.; Gagne, R. R.; Reed, C.; Halbert, T. R.; Lang, G.; Robinson, W. T. Picket fence porphyrins. Synthetic models for oxygen binding hemoproteins. J. Am. Chem. Soc. 1975, 97, 1427-1439.

(74) Mispelter, J.; Momenteau, M.; Lhoste, J. Proton magnetic resonance in fourcoordinated ferrous porphyrins. Mol. Phys. 1977, 33, 1715-1728.

(75) Jameson, G.; Robinson, W. T.; Collman, J. P.; Sorrell, T. N. Crystal and molecular structure of a novel, high-spin, iron(II), "picket fence" por- 
phyrin derivative. catena- $\{\mu$-[meso-Tetrabis $(\alpha, \alpha, \alpha, \alpha$-o-pivalamidophenyl)porphinatoN,N,N",N"':O]-aquoiron(II)-tetrahydrothiophene\}. Inorg. Chem. 1978, 17, 858-864.

(76) Mispelter, J.; Momenteau, M.; Lhoste, J. Proton magnetic resonance characterization of the intermediate $(\mathrm{S}=1)$ spin state of ferrous porphyrins. J. Chem. Phys. 1980, 72, 1003-1012.

(77) Aquilante, F.; Autschbach, J.; Carlson, R. K.; Chibotaru, L. F.; Delcey, M. G.; De Vico, L.; Fdez. Galván, I.; Ferre, N.; Frutos, L. M.; Gagliardi, L. Molcas 8: New capabilities for multiconfigurational quantum chemical calculations across the periodic table. J. Comput. Chem. 2016, 37, 506-541.

(78) Liao, M.-S.; Scheiner, S. Electronic structure and bonding in metal porphyrins, metal= Fe, Co, Ni, Cu, Zn. J. Chem. Phys. 2002, 117, 205-219.

(79) Pinjari, R. V.; Delcey, M. G.; Guo, M.; Odelius, M.; Lundberg, M. Cost and sensitivity of restricted active-space calculations of metal L-edge X-ray absorption spectra. J. Comput. Chem. 2016, 37, 477-486.

(80) Forsberg, N.; Malmqvist, P.-A. Multiconfiguration perturbation theory with imaginary level shift. Chem. Phys. Lett. 1997, 274, 196-204.

(81) Ghigo, G.; Roos, B. O.; Malmqvist, P.-Å. A modified definition of the zeroth-order Hamiltonian in multiconfigurational perturbation theory (CASPT2). Chem. Phys. Lett. 2004, 396, 142-149.

(82) Vancoillie, S.; Zhao, H.; Radon, M.; Pierloot, K. Performance of CASPT2 and DFT for relative spin-state energetics of heme models. J. Chem. Theory. Comput. 2010, 6, $576-582$.

(83) Zobel, J. P.; Nogueira, J. J.; González, L. The IPEA dilemma in CASPT2. Chem. Sci. 2017, 8, 1482-1499. 
(84) Douglas, M.; Kroll, N. M. Quantum electrodynamical corrections to the fine structure of helium. Ann. Phys. 1974, 82, 89-155.

(85) Hess, B. A. Relativistic electronic-structure calculations employing a two-component no-pair formalism with external-field projection operators. Phys. Rev. A 1986, 33, 3742.

(86) Roos, B. O.; Lindh, R.; Malmqvist, P.-A.; Veryazov, V.; Widmark, P.-O. Main group atoms and dimers studied with a new relativistic ANO basis set. J. Phys. Chem. A 2004, 108, 2851-2858.

(87) Roos, B. O.; Lindh, R.; Malmqvist, P.-A.; Veryazov, V.; Widmark, P.-O. New relativistic ANO basis sets for transition metal atoms. J. Phys. Chem. A 2005, 109, 6575-6579.

(88) Boström, J.; Delcey, M. G.; Aquilante, F.; Serrano-Andrés, L.; Pedersen, T. B.; Lindh, R. Calibration of Cholesky auxiliary basis sets for multiconfigurational perturbation theory calculations of excitation energies. J. Chem. Theory. Comput. 2010, $6,747-754$

(89) Malmqvist, P.-A.; Roos, B. O. The CASSCF state interaction method. Chem. Phys. Lett. 1989, 155, 189-194.

(90) Malmqvist, P.-A.; Roos, B. O.; Schimmelpfennig, B. The restricted active space (RAS) state interaction approach with spin-orbit coupling. Chem. Phys. Lett. 2002, 357, 230240.

(91) Guo, M.; Källman, E.; Sørensen, L. K.; Delcey, M. G.; Pinjari, R. V.; Lundberg, M. Molecular orbital simulations of metal 1s2p resonant inelastic X-ray scattering. J. Phys. Chem. A 2016, 120, 5848-5855.

(92) Lang, G.; Spartalian, K.; Reed, C. A.; Collman, J. P. Mössbauer effect study of the magnetic properties of $\mathrm{S}=1$ ferrous tetraphenylporphyrin. J. Chem. Phys. 1978, 69, $5424-5427$. 
(93) Boyd, P. D.; Buckingham, D. A.; McMeeking, R. F.; Mitra, S. Paramagnetic anisotropy, average magnetic susceptibility, and electronic structure of intermediate-spin $\mathrm{S}=1$ (5,10,15,20-tetraphenylporphyrin)iron(II). Inorg. Chem. 1979, 18, 3585-3591.

(94) Ikeno, H.; Tanaka, I.; Koyama, Y.; Mizoguchi, T.; Ogasawara, K. First-principles multielectron calculations of $\mathrm{Ni}_{2,3}$ NEXAFS and ELNES for LiNiO2 and related compounds. Phys. Rev. B 2005, 72, 075123.

(95) Kadek, M.; Konecny, L.; Gao, B.; Repisky, M.; Ruud, K. X-ray absorption resonances near $\mathrm{L}_{2,3}$-edges from real-time propagation of the Dirac-Kohn-Sham density matrix. Phys. Chem. Chem. Phys. 2015, 17, 22566-22570.

(96) Huse, N.; Kim, T. K.; Jamula, L.; McCusker, J. K.; de Groot, F. M.; Schoenlein, R. W. Photo-induced spin-state conversion in solvated transition metal complexes probed via time-resolved soft X-ray spectroscopy. J. Am. Chem. Soc. 2010, 132, 6809-6816.

(97) Guo, M.; Sørensen, L. K.; Delcey, M. G.; Pinjari, R. V.; Lundberg, M. Simulations of iron K pre-edge X-ray absorption spectra using the restricted active space method. Phys. Chem. Chem. Phys. 2016, 18, 3250-3259.

(98) Sørensen, L. K.; Guo, M.; Lindh, R.; Lundberg, M. Applications to metal K preedges of transition metal dimers illustrate the approximate origin independence for the intensities in the length representation. Mol. Phys. 2017, 115, 174-189. 


\section{Graphical TOC}

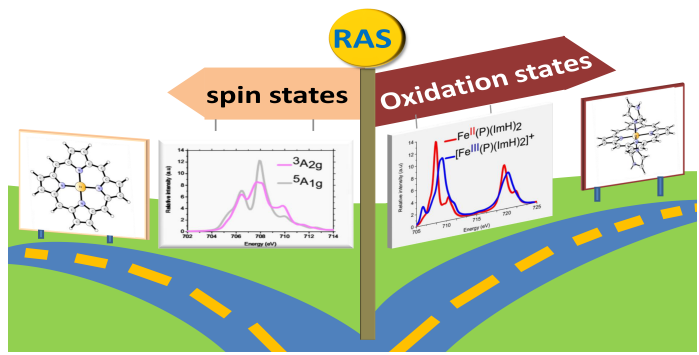

